\title{
Fusion of medical images in wavelet domain: an algorithmic model
}

Fusión de imágenes médicas en dominio wavelet: un modelo algorítmico Fusão de imagens médicas no domínio wavelet: um modelo algorímico

\section{Satya Prakash Yadav ${ }^{1}$ Sachin Yadav ${ }^{2}$}

Received: September $9^{\text {th }}, 2020$

Accepted: November $19^{\text {th }}, 2020$

Available: January 11th, 2021

Howto cite this article: S.P. Yadav, S. Yadav,"Fusion of Medical Images in Wavelet Domain: an Algorithmic Model," Revista Ingeniería Solidaria, vol. 17, no. 1, 2021, doi: https://doi.org/10.16925/2357-6014.2021.01.07

Research article. https://doi.org/10.16925/2357-6014.2021.01.07

1 Department of Information Technology, ABES Institute of Technology (ABESIT), Ghaziabad-201009, India,

Email: prakashyadav.satya@gmail.com,satya.yadav@abesit.in

Orcid: https://orcid.org/0000-0002-2634-5600

2 Department of Computer Science and Engineering, G.L. Bajaj Institute of technology and Management (GLBITM), Greater Noida- 201306, India,

Email: sachin.yadav@glbitm.org

Orcid: https://orcid.org/0000-0003-2432-3544 


\section{Abstract}

Introduction:Image Fusion techniquesconsist of three stages: extraction of features, reduction of dimensions, and classification.

Problem:This paper presents a novel approach for Multiresolution analysis.

Objective: It is most widely used in image fusion science, which captures the features of an image not only at different resolutions, but also at different orientations.

Methodology:This Wavelet based algorithm has additional advantages of fast implementation, versatility, auxiliary memory saving, complete reconstruction properties and simplicity as wavelet transformation was used.

Results: The simulation results of the MRI and CT images show perfectly acceptable image quality and cover disease detection in the subsequent final image.

Conclusion: Principal Component Analysis (PCA) based on fusion algorithms will empower medical researchers or clinicians to properly apply image fusion and data transmission, which leads to better care practices to minimize redundancies and can also handle data loss.

Originality:Fusing images can decrease the image size, which can decrease the bandwidth when transmitting images. This also compresses the images; here an attempt is made to retain the same consistency.

Limitations:As this is still a relatively novel method, mistakes with regards to the handling of clinical data may prompt treatment deficiencies for the patient. Image quality must not be diminished with this usage.

Keywords: Fused Image, Fusion Algorithms, Principal Component Analysis, ICA, Wavelet Transformation, Image fusion.

\section{Resumen}

Introducción: Las técnicas de Image Fusion constan de tres etapas: extracción de características, reducción de dimensiones y clasificación.

Problema: Este artículo presenta un enfoque novedoso para el análisis multirresolución.

Objetivo: Es más utilizado en la ciencia de fusión de imágenes, que captura las características de una imagen no solo en diferentes resoluciones, sino también en diferentes orientaciones.

Metodología: Este algoritmo basado en Wavelet tiene ventajas adicionales de implementación rápida, versatilidad, ahorro de memoria auxiliar, propiedades de reconstrucción completa y simplicidad ya que se utilizó la transformación de wavelet.

Resultados: Los resultados de la simulación de las imágenes de resonancia magnética y tomografía computarizada muestran una calidad de imagen perfectamente aceptable y cubren la detección de enfermedades en la imagen final posterior.

Conclusión: El análisis de componentes principales (PCA) basado en algoritmos de fusión permitirá a los investigadores médicos o clínicos aplicar correctamente la fusión de imágenes y la transmisión de datos, lo que conduce a mejores prácticas de atención para minimizar las redundancias y también puede manejar la pérdida de datos.

Originalidad: la fusión de imágenes puede disminuir el tamaño de la imagen, lo que puede disminuir el ancho de banda al transmitir imágenes. Esto también comprime las imágenes; aquí se intenta mantener la misma consistencia. 
Limitaciones: como se trata de un método relativamente nuevo, los errores en el manejo de los datos clínicos pueden provocar deficiencias en el tratamiento del paciente. La calidad de la imagen no debe disminuir con este uso.

Palabras clave: imagen fusionada, algoritmos de fusión, análisis de componentes principales, ICA, transformación de ondas, fusión de imágenes.

\section{Resumo}

Introdução: as técnicas de fusão de imagens consistem em três estágios: extração de recursos, redução de dimensões e classificação.

Problema: Este artigo apresenta uma nova abordagem para análise de multirresolução.

Objetivo: é mais amplamente utilizado na ciência de fusão de imagens, que captura as características de uma imagem não apenas em diferentes resoluções, mas também em diferentes orientações.

Metodologia: Este algoritmo baseado em Wavelet tem vantagens adicionais de implementação rápida, versatilidade, economia de memória auxiliar, propriedades de reconstrução completa e simplicidade quando a transformação de wavelet foi usada.

Resultados: Os resultados da simulação das imagens de ressonância magnética e tomografia computadorizada mostram uma qualidade de imagem perfeitamente aceitável e abrangem a detecção de doenças na imagem final subsequente.

Conclusão: a análise de componentes principais (PCA) baseada em algoritmos de fusão capacitará os pesquisadores médicos ou clínicos a aplicar adequadamente a fusão de imagens e a transmissão de dados, o que leva a melhores práticas de atendimento para minimizar redundâncias e também pode lidar com a perda de dados.

Originalidade: a fusão de imagens pode diminuir o tamanho da imagem, o que pode diminuir a largura de banda ao transmitir imagens. Isso também comprime as imagens; aqui é feita uma tentativa de manter a mesma consistência.

Limitações: Por se tratar de um método ainda relativamente novo, erros no manuseio dos dados clínicos podem levar a deficiências no tratamento do paciente. A qualidade da imagem não deve ser diminuída com esse uso.

Palavras-chave: Fused Image, Fusion Algorithms, Principal Component Analysis, ICA, Wavelet Transformation, Image fusion.

\section{Introduction}

Medical imaging takes on a significant role in various uses of therapeutic research and medical therapy technological advancement in the present period. It involves progressively greater pictures with much-improved properties and data to identify and diagnose the health image accurately. Therefore, the merger of medical pictures offers the best answer to the high quality and redundant collection of image data. Multi-modal fusion of medical images increases subsequent image quality 
spontaneously afterward fusion. The image-fusion requires a minimum of two explicative images and generates additional data to better represent the final image produced. The resulting image also contains the corresponding and redundant data.

Medical imaging is, in several respects, an essential aspect of routine clinical use. The presence of distinguishing imaging methods to show system anatomy in different human body parts is widely appreciated. Modalities such as MRI and Computer Tomography (CT) provide fewer and mutual information [1] [2]. For example, CT scans offer data about solid tissues, MRI scans give better data about accessible tissue, PET images provide high-resolution images, which generates an enormous need for the fusion of images to obtain relevant data from fused pictures. The fusion of these images individually produces favored integrated details, which in the bio laboratories shows a distinctive structure.

For example, WT, principal component analysis (PCA), an independent investigation of components (ICA) with strategies categorized by spatial method, and the High Pass Filtration Technology are other critical areas of fusion strategy for image fusion purposes [3] [4]. Resulting images provide improved image elements and facilitate the recognition, interpretation, and identification of references [5]. The application of a set of image functions results in the selection of actual data in a single image. This isunderlined in the final picture [6]. The resulting images are fused by combining the best-amplified data from the first images in a final fused image.

\subsection{Fusion of Medical Images}

Image Fusion incorporates and transforms image information from at least two input images into one image to improve image quality and sound and reduce distortion in the final image. The second-generation wavelet domain provides a method for the image fusion of multi-modal images [7]; a technique of fusion that is suitable for unsurpassed non-coincident collected data. Decision-level fusion is most-ideal for these results, but in principle is not ideal as targets not identified by all sensors do not benefit from fusion in their full capacity [8]. Uniqueness of image preparation requires high spatial and exclusive data for unrivaled image quality in a fused image.

Image fusion will address relevant questions of image quality as a combination method that collects and re-generates all the necessary information from multiple images in a blended model called a mixed (fused) image of higher quality and magnitude. Medical images have unique qualities of space in a medical setting that differ from image to picture. These characteristics can be described as: image heterogeneity. Health pictures refer to living creatures, pieces of the body, and tissue. The internal 
structure, shape, and size of these parts may vary from patient to patient from a few perceptions of a particular patient, regardless that they are obtained with the same technology and comply with standard procurement law.

\subsection{Problem Statements}

For relative analyses, various approaches have been reviewed, and we can provide our argumentsfor or against the strategies used for photo merger from previous studies. The basic standard technique generates unwanted signs, such as decreased comparisons, while a standard weighted procedure involves a user modulated weight measurement threshold. Most possibly, a few plans propose high spatial quality but suffer from spectral distortion deficiencies, notably:

- Simple wavelet theory.

- Wavelet generalizations and extensions, like wavelet techniques included in the refining stage.

- In applications with functional data analysis, for instance, discrete timeseries data sampling may be necessary for review, the wavelet techniques were not extensively performed.

\section{Related methods and scope}

Across three steps, the fusion process primarily excluded: pixel, Function level, and level of judgment. Rate of Pixel Fusion is the basis for the fusion of pictures of pixels recovered from the base picture sequence. This method of synthesis uses the field of space or occurrence to minimize contrast [2].

Fused Feature is the edge and limit input image information extracted from the maximum criterion of the wavelet transform module. Standard parameters of deviation, entropy, interentropy, and gradient are evaluated for image fusion efficiency and with better image fusion results being produced by the proposed process, as image contrast, average information quality, and accurate fused image information are increased. [7].

CT and MR fused pictures allow the Simultaneous visualization of MR CT anatomy and soft tissue anatomy images to diagnose the ailment accurately to make interventions more effective [13]. 


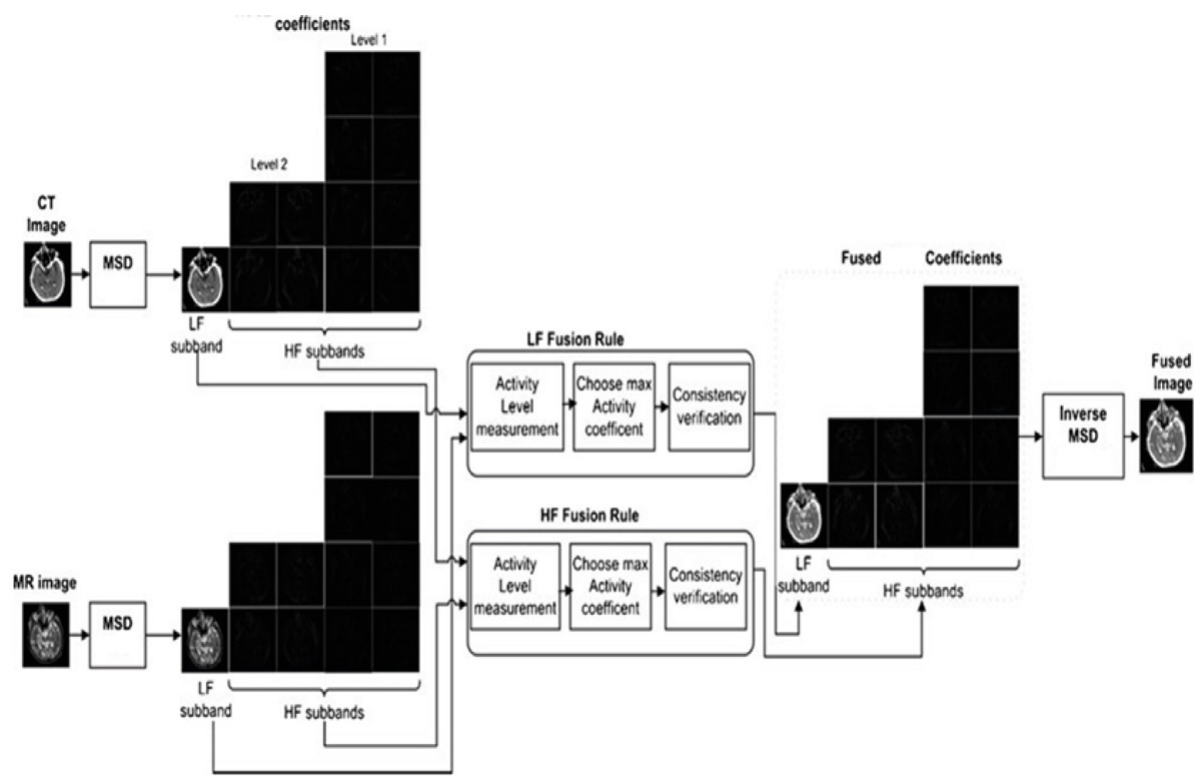

Figure. 1. Fusion of Multi Scale Images

Source: [13]

CT-MRI Present a model-based probabilistic approach to the fusion of multisensory images that address the weakness of existing solutions. Here the following Fusion function is formulated as a question for the estimation of the actual sensor picture underlying scene. We model the sensor images as the noisy functions of this real scene locally [4]. The parameters of a function specifically include the reversal of the local contrast and the presence in the sensor images of sensor-specific data.

After wavelet transformation and the gray fusion stage, a product of Anatomical and systemic improvements infuse details into the region of gray material (GM) and manipulate the white matter spectral field information in the brain.

Typically, the collection of Binary brain imaging data is now available from one subject that is popular for physical imaging research [8]. Numerous mutually enhanced and efficient and easy techniques for blind source separation, which has advantages over the multi-task fusion of data between ICA (Independent Component Analysis) and CCA (Canonical Correlation Analysis). The ICA only focuses on the localization features other than offering pyramid decomposition or a multi-level representation as wavelets. This has been the main reason for the growing importance of data-driven methods, and in particular of independent component analysis (ICA) as it provides useful decompositions with a simple generative model and using only 
the assumption of statistical independence where Canonical Correlation Analysis (CCA) is a statistical method that summarizes the correlation structure between two random vectors by linear transformations.CCA has the advantage that the source separation performance improves as the number of datasets increase. The method outperforms the existing group analysis methods in jointly separating groups of corresponding sources with heterogeneous correlation values or, in the case of complex-valued data, when sources have circular and non-circular distributions. So, Fusion of Multi Scale Images provides suitable Connections and sturdy precision to estimate these two data sets, where the basis has different or specific correlations between data sets. The task-related activation is expressed by a "simple" and is more efficient than a four-dimensional data set with condensed data, because of its compact dimensions.

\section{Literature review}

Various imaging methods, including pixels, pyramids, wavelets, contour lets, etc. have been studied in several studies. In different areas, the techniques of image fusion come in various dimensions.

Many fusion algorithms have been introduced in previous years to increase the standard image fusion algorithms, such as maximum and minimum collection patterns [9], simple average and weight averages, etc. Though, the significant advancement of inquiries in this field has led to the use of algorithms such as Wavelet Transformation [10], PCA and HIS [11] in the fusion of medical images.

Besides, real-valued wavelet transformations have previously been used for fusion images. This technique has improved over more popular methods; the conversion is affected by the change variation and lack of a wavelet base directionality. The reversible and discreet complex Wavelet transforms (DT-CWT Transform Double Tree Complex) solve these problems. [12] Introduces a more versatile, highly directional, and shift-invariant alternative structure such as DTCWT, which outperforms the traditional PSNR method and improves image quality.

In [13], the author implemented image fusion algorithms focused on wavelets to merge images based on low-light double coverage. This technique used conventional methods for wavelet disintegration and a mechanism for maximizing the target contrast to fuse pyramids. The objective distinction was measured by the relation between the wavelet coefficient as well as local luminosity over a template of $5 \times 5$. Along with waves optimised for image fusion, pixel level methodology is the most 
exciting high strength [14]. However, the blending, as mentioned earlier, produced a highly focused picture; the picture was bright and had reduced contrasts. The work addressed in [15] focused on the analysis of the DWT.

\section{Image fusion algorithms}

Fusion images may use multi-modal, multi-temporal, or multi-sensor images. Therefore, the best approach for the fusion of images needs to take those essential factors into account:

I. Fusion of medical pictures no longer adds unnecessary objects that can contribute to misinterpretation.

II. The fused description conserves all the necessary information from the original images.

The foremost necessary step to enable the image fusion process is picture registration. The registration of images is the way to transform disparate knowledge arrangements into an incentive system.

The algorithms of the combination of images can be categorized into various dimensions: pixels, transformers as well as features. A fusion for pixel-level is utilized directly on the input image and its mechanism on the features derived from the original images. The approaches to image fusion, such as the weighting, simple maxima/ minima, PCA and IHS, are spatial domain techniques.

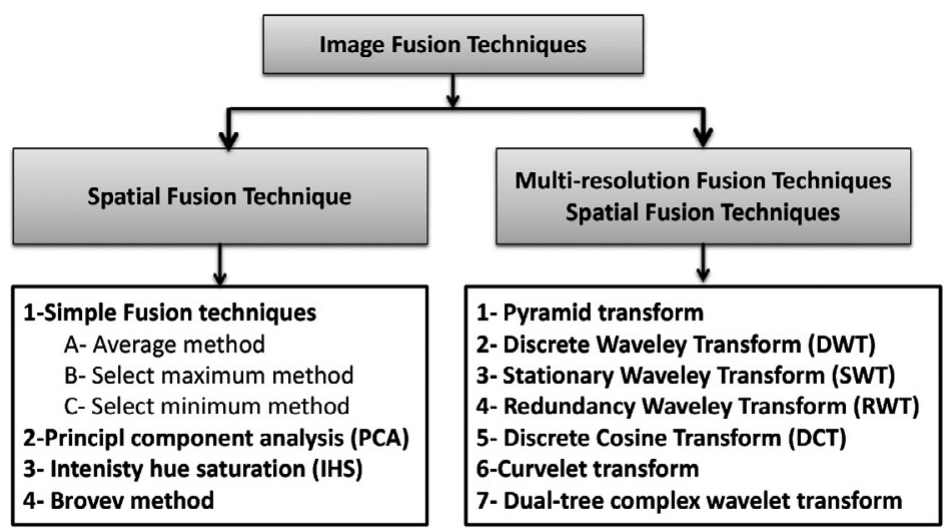

Figure 2. Popular Image fusion techniques Source: own work 
The transform domain section allocated to fusion processes such as WT, DWT, and DCT. Figure 3 shows the use of these approaches for data fusion.

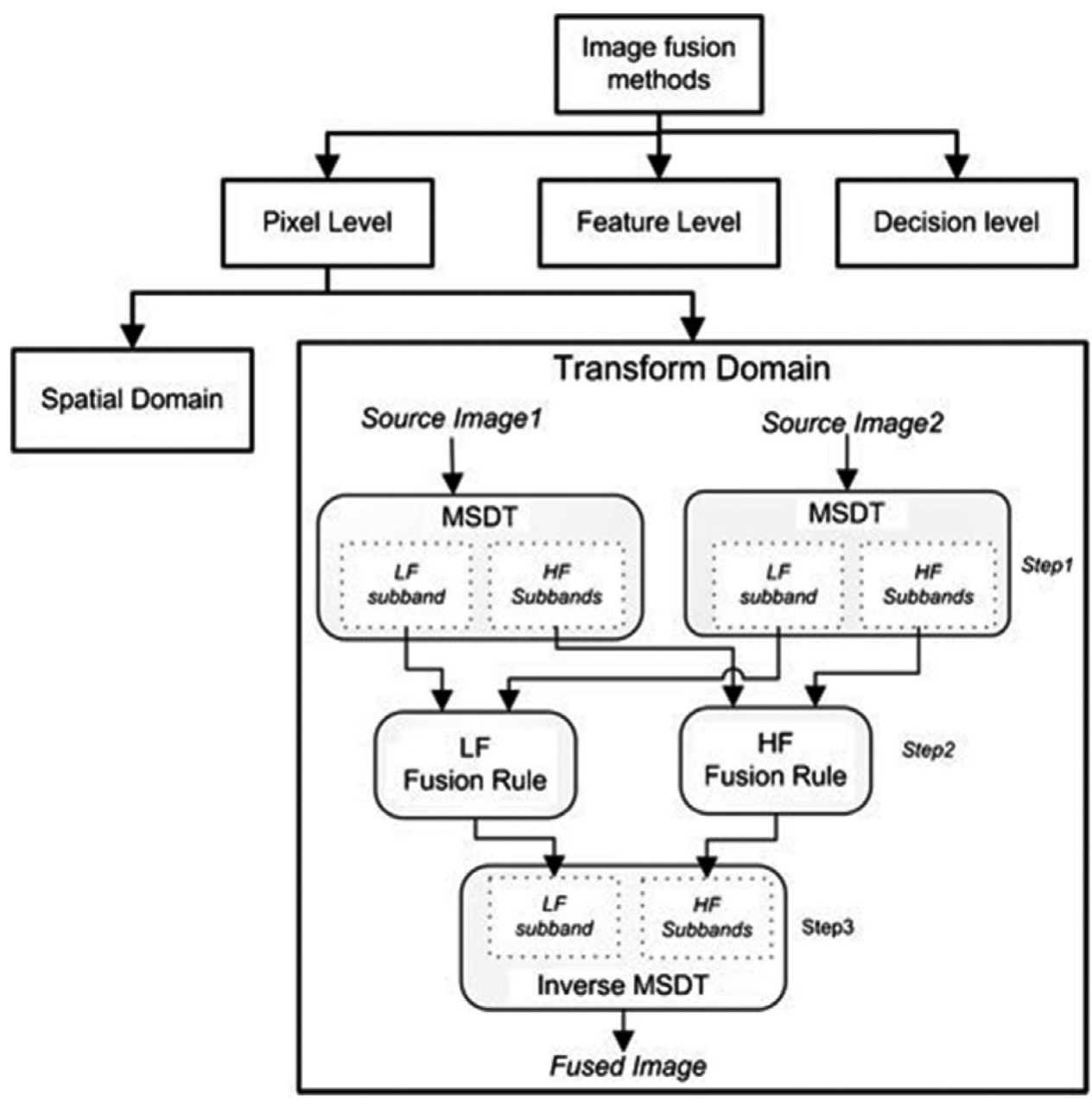

Figure 3. Multi-level fusion system in all three processing Source: [13]

The picture has the property of multi-scale or multi-resolution expansion in two dimensions. Then image extension and compression are used to in the scaling process to retrieve the specific frequency data that can be separated into four quarter-sized images.

\subsection{Basic components}


Each segment describes the various essential components for the fusion of images.

Figure 2 displays the classification of different fusion of image technique rates.

1. Average Pixel Intensity

$\mathrm{F}$ is indicating the Average Pixel Intensity; see Eqn. (1) below.

$$
\bar{F}=\frac{\sum_{i^{*}} \sum_{j}(f(i, j)}{m^{*} n}
$$

\section{Standard Deviation (SD)}

The root mean square of the variance measures the average variance. It applies in the case of data distribution; the standard deviation (SD) from noise and signal components usually achieved. Nevertheless, this calculation increases the effectiveness without considering the noise components. More disseminated pictures of knowledge should have a higher SD by [16].

$$
S D=\sqrt{\frac{\sum_{i^{*}} \sum_{j}(f(i, j)-\bar{F})^{2}}{m^{*} n}}
$$

\section{Entropy $(\mathrm{H})$}

Entropy is an aspect used to measure image information quality. Lower entropy values mean increases in data and improvements in fusion efficiency. But noise and other unwanted regular displacements are erogenous. The estimation of entropy can be achieved by [17] [18].

$$
E=\sum_{i=0}^{L-1} P_{i} \log _{2} P_{i}
$$

When $L$ is the summation of gray and pi shows the relationship among the integer of l-gray pixels $(0<\mathrm{l}<\mathrm{L}-1)$ as well as the over-all pixel count found in image; in other words, pi is the normalized probability of every Gray Level.

\section{Spatial Frequency (SF)}

SF is utilized to calculate the complete operation and precision of the images. The significant value of SF indicates a more robust fusion outcome [19]. 


$$
S F=\sqrt{R F^{2}+C F^{2}}
$$

RF and CF are the row and column frequency respectively,

$$
R F=\frac{1}{M(N-1)} \sum_{i=0}^{M-1} \sum_{j=0}^{N-2}(F(i, j+1)-F(i, j))^{2}
$$

and

$$
C F=\frac{1}{(M-1) N} \sum_{i=0}^{M-2} \sum_{j=0}^{N-1}(F(i+1, j)-F(i, j))^{2}
$$

\subsection{Discrete Cosine Transform}

DCT efficiency is higher than the Discrete Fourier Transformation. The condensed histogram attained by the DCT is, therefore, fewer clustered than the DFT phase when the amplitude-specific range of the picture contrasted with the DCT diagram and the DFT [20]. Energy compression is essential because image compaction is advantageous in other applications.

\subsection{Region Based Method}

Initially, Yang and Li depicted this approach and provided a regional guideline on the spatial fusion of images [21]. At this stage, two techniques are proposed based on the image fusion region. They devised a combination strategy using large blocks centered on a geographical area in the field. The algorithm for spatial areas in regional fusions, however, is conceptual and can be used in real-time using settled size blocks. In practical experiments, it is equally necessary. The results of several studies are nevertheless aspiring; additional factors should be taken into account in contrast to MRI images.

\subsection{Principal Components Analysis Method}


The PCA is a numerical tool that helps break down different components and reduce them to various parts. For the PCA image merger, the covariance condition of the 2 input images is the central concept. Commonly speaking, PCA uses all pixel image parameters for each pixel range, [23] adding a common element to each pixel rate to the mean pixel-values so that the final image outcome is produced on a single-pixel scale. This technique calls for the coding and implementation of computer programs to select primary segments from all regions of the picture. Weighted coefficients (WC) collected via Eigen's vectors and values. The weighted coefficients method allows the generation of an ideal fused image. The PCA strategy controls the weighted components perfectly. The unnecessary noise elements can be the use of the PCA action method for fusion of representation to achieve superior image quality.

The PCA is a less complex and demonstrated self-vector analysis method. It requires identification and data design courses to explain parallels and comparisons and thus reduces the estimation of redundancy without data loss. The vectors in this method are first isolated from unique data structure designated images and covariance function resolute. Every element of the vector covariance corner contains dissimilarities of each vector part. The cross-sectional estimates of covariance in Eigen Values and Eigen Vectors are analyzed.

If the two images input from $\mathrm{G} 1(\mathrm{x}, \mathrm{y})$ and $\mathrm{G} 2(\mathrm{x}, \mathrm{y})$ should be attached into column vectors, $n \times 2$ dimensions will be their vector and $n$ vector for each vector length of the image. This describes the fused picture (Fig. 4 shows the flowchart):

$$
\mathrm{G}_{\mathrm{f}}(x, y)=P_{1} G_{1}(x, y)+P_{2} G_{2}(x, y) \quad 5
$$




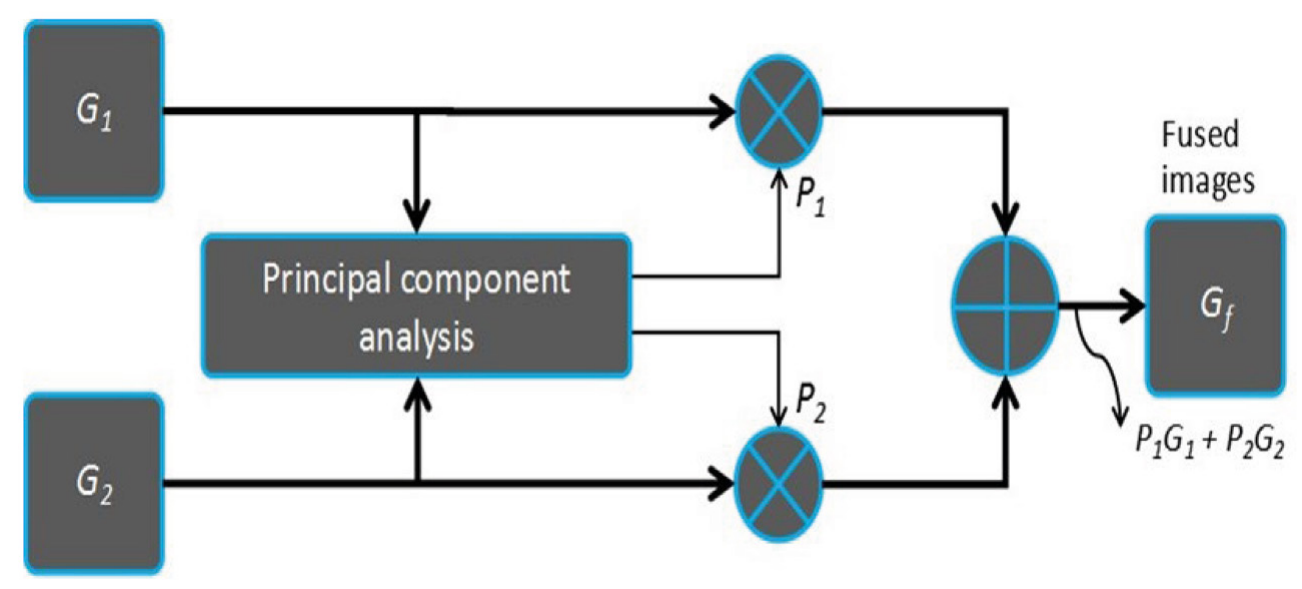

Figure 4. Diagram of flow of information in PCA

Source: own work

\subsection{ICA Method}

In [23], the expertise of different engineered methods of transformation using ICA and ICA (t-ICA) projected in their investigation of image fusion was demonstrated. The complementary bases are made of images from parallel configurations to visual aspects in offline mode. In the transmute arena, the images combined to reflect new guidelines for regional fusions. Therefore, uncontrolled variation, according to the ICA image fusion technique, is presented. The forwarded scheme featured better performance as compared to wavelet-dependent methodologies and a slightly expanded device complexity. In their study, the researchers made use of both regionally centered and ICA for image fusion.

\subsection{Wavelet Transform Method}

Wavelets are able to select and manipulate the frequencies in both time and space and, therefore, extract the quality of detailed information that is stored in the high frequency in images. Wavelets have many known applications in medical image fusion such as image pseudo coloring, medical diagnosis, feature level image fusion, medical segmentation, super resolution, lifting scheme, 3D conformal radiation therapy treatment planning and color visualization [24] where the Fourier transform is a useful tool to analyze the frequency components of the signal. The WT is a form of FT that is developed for the appropriate selection of temporary Fourier changes. 
In image processing applications, wavelets are used due to simultaneous time and frequency analysis, where Fourier only does frequency analysis. The leading advantage of utilizing the WT is that the standard 2D images are disintegrated into various resolutions. The WT based image fusion can be categorized into various groups, for example, bi-orthogonal as well as orthogonal, etc. Although, Wavelet has a unique characteristic for decompression and propagation, these wavelets exchange a few specific components, resulting in detailed fusions [25][26]. WT is commonly used in image fusion, edge detection, and shape analysis, and data compression.

The earliest and most important, according to the WT image grouping, was established in [12] as well as [27] and utilized the method to decompose input images and their designated features for region-based as well as pyramidal fusions. Despite the fact that this technique works best in subordinate pyramids, bulk filtering for broad areas will sway choices to any information source for a more elegant emphasis.

\subsubsection{Families in Wavelets}

Several vital operations are utilized as the substantial primary WT's mother. Indeed, it is the primary Wavelet that uses the scale and rendering process to create all wavelets used in transformation. Therefore, the application should take into consideration the fine details, and an applicable mother wavelet was chosen so that the wavelets transform effectively. A portion of the normal wavelet functions are shown in Figure 5.

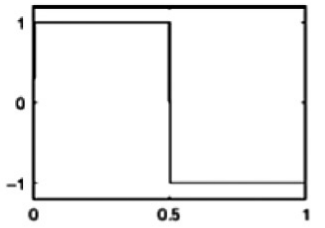

(a)

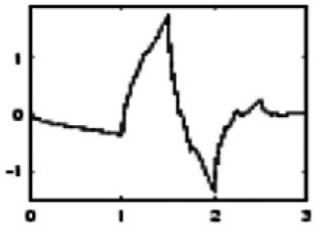

(b)

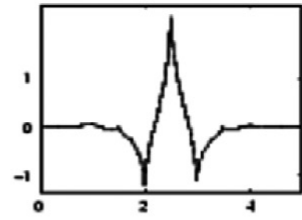

(c)

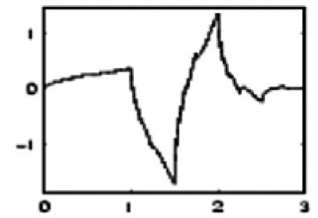

(d)

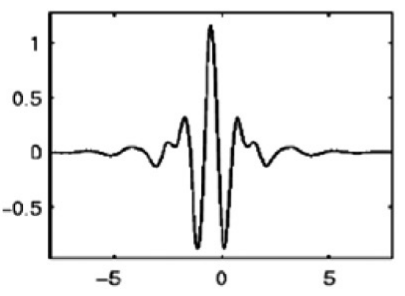

(e)

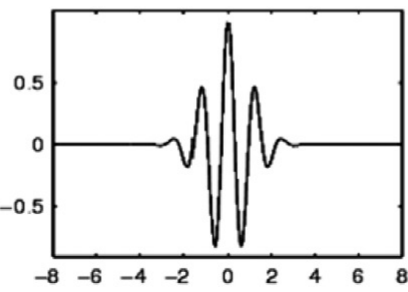

(f)

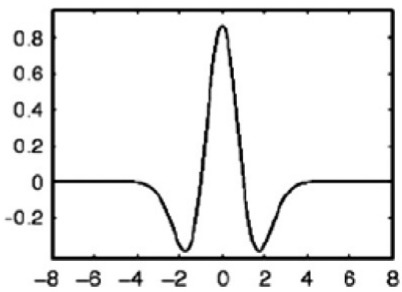

(g)

Figure 5. Different Wavelets- a) Haar b) Daubechies c) Coiflet d) Symlet e) Meyer f) Morlet g) Mexican Hat Source: own work 
This Wavelet allows us to detect waves in the time or frequency domain and decompose the final energy signal into a wavelet function size. The WT is a process where two or more images are distributed into an image, remembering the crucial features of every source image [11].

\subsection{Proposed Algorithm}

The current step of the image fusion proposed algorithm includes the fusion rule of Photos of MRI and CT with the wavelet decomposition. WT is a secure tool for removing images at different resolution levels. This technique requires significant storage and is more costly computationally. The PCA was used to reduce the aspect of and increase the vector power of discrimination. The first module inquiry was used to provide a more precise and speedy solution than traditional methods for the classification of brain tumors.

$\mathrm{K}-$ Nearest Neighbor $(\mathrm{K}-\mathrm{NN})$ is regarded as a revolutionary classifier and is capable of producing top results in complex applications at any time. In educational accumulation, the K-NN uses a division of purposes to make sense of which records a collection spot has. A social occasion is confined when the partition inside the data is near, and when the separation within the information is far away, with different conversations modeled. In MRI and CT studies, K-NN is commonly used as a designation for collecting images of the bodies. K-NN was recycled, for example, to arrange epileptic and traditional personality behaviors using MRI and CT images. Then findings of the comparison of classifications of medical images using PCA are addressed with the majority of nearest substances for the application of brainwave balancing.

Here we offer a wavelet transformation algorithm using PCA which combines the benefits of the algorithms described:

Start

\{

Stage 1: The Approximate coefficients extracted using db3 wavelet transform (DWT) up to the third decomposition level.

Stage 2: Use PCA, leading to efficiency in approach for results in a classifier that is more effective and accurate.

Stage 3: Select the number of nearest neighbors and determine the appropriate distance metric. 
Stage 4: In the distance matric: Save all the training data collection $S$ in pairs (according to the features selected) $S=\{(x i, d i), i=1 \ldots n\}$, where $x i$ is a pattern of training distance metric, di.

Stage 5: Calculate the distance between the new vector and all stored characteristics (distance metric, di).

Stage 6: The nearest neighbors are picked and the new item placed in the class with the greatest number of close items.

Stage 7: Output: Fused image.

\}

Finish

The first step of the suggested fusion process involves the precursor of CT or MRI images, which is to convert the image from RGB to grayscale (RGB segments of images converted into sections of grayscale). The next step is to analyze the photos used to WT (Figure 6). The WT turns the image into frequency groups, notably a lower repeating group and other higher repetitive groups[29]. Furthermore, wavelets referred to as the fusion of two scaling operations: $\psi(\mathrm{t})$, primary wavelet function often called the mama-wavelet (father-wavelet), and secondary wavelet function. Mother wavelet adjustments and scaling to generate wavelet units are similar to the following:

$$
\psi_{a, b}(t)=\frac{1}{\sqrt{a}} \psi\left(\frac{t-b}{a}\right) \text { for }(a, b \varepsilon R), a>0
$$

Where; $\psi(\mathrm{t})$ is the mother-wavelet function, while $\mathrm{a}, \mathrm{b}$ are the particular parameters of scale and translation, as in the Equation below.

$$
a=a_{o}^{j}, b=m a_{o}^{j} b_{o} \text { for }(j, m \varepsilon Z)
$$

Thus, the wavelet family characterized by Equation below:

$$
\psi_{j, m}(t)=a_{o}^{-j / 2} \psi\left(a_{o}^{-j} t-m b_{o}\right) \text { for }(j, m \varepsilon Z) \quad 8
$$

\subsection{The Root Mean Square Error}

The Root Mean Square Error (RMSE) is a generally utilized reference-according to evaluation metric. The RMSE of a reference image, $R$, and the fusion image $F$, is shown in the following state: 


$$
R M S E=\sqrt{\frac{1}{M N}} \sum_{i=1}^{M} \sum_{j=1}^{M}(R(i, j)-F(i, j))^{2}
$$

When $R(I, j)$ and $F(I, j)$ control and fuse images, the images' attributes are independent, and $\mathrm{M}$ and $\mathrm{N}$ are keeping the RMSE value low, improving efficiency and performance.

\section{Experiments and Results}

There are experimentations conducted with MATLAB (Figure 6) besides the outcomes obtained. The brain CT and MRI scans aim to construct three datasets. The images are split horizontally and vertically reflecting the Wavelet first-state transformation so that the pictures are divided into Parts $H H, L H, H L$, and LL (see Figure 7).

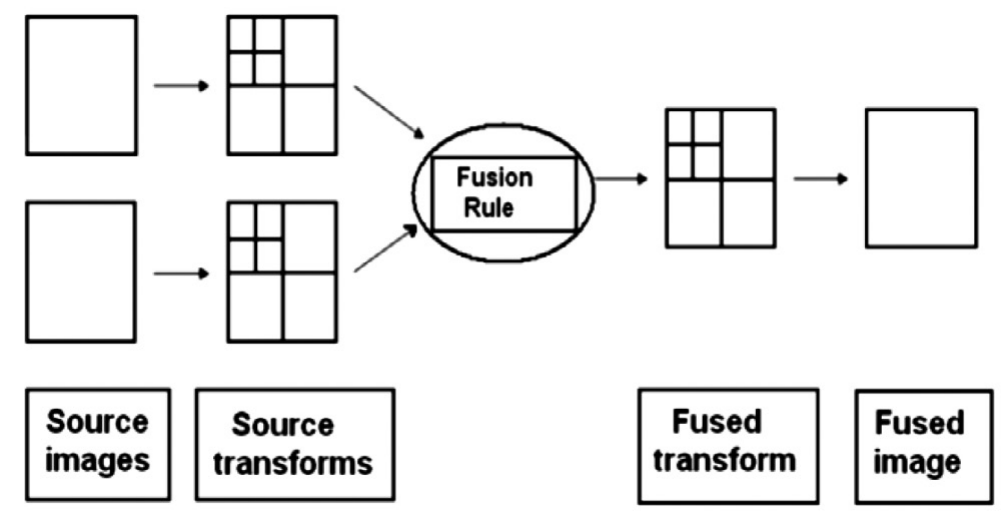

Fig 6. Diagram of wavelet-based image disintegration

Figure 7 shows the concept of a discrete wavelet transform (DWT). A discrete Wavelet Transform is computed by changing the scale of the analysis window each time, shifting it or multiplying it by the signal. Wavelet Transform is represented by the connection between the signal and wavelet at different scales (frequency inverse) and the signal. 


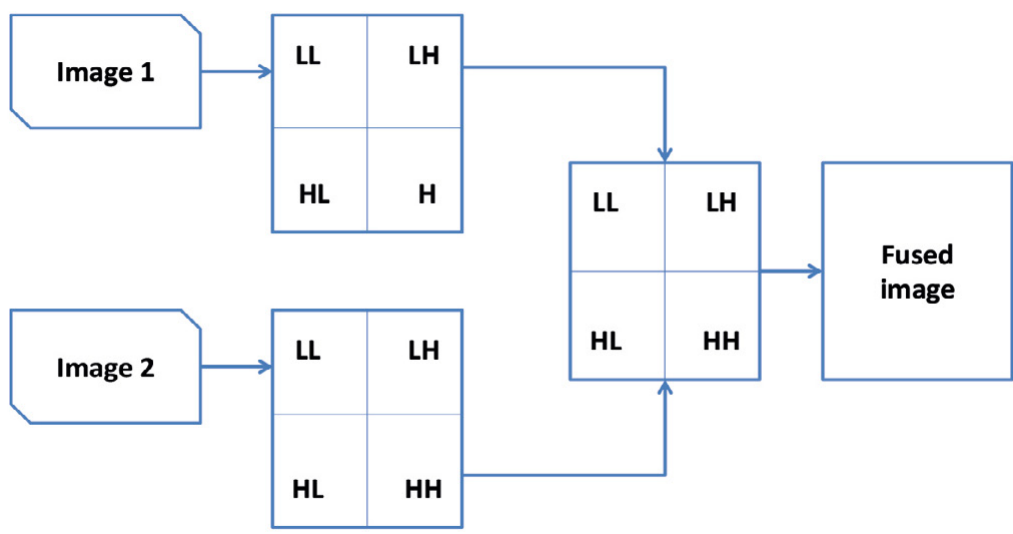

Figure 7. Scheme of discrete wavelet transforms

Source: own work

All image formats have to be completed before being transmitted by image alteration, sub-band Quantization, and coding. To increase the visible information in the picture with the transformation of waves and usage of the PCA and K-NN image de-noising methods, this strategy proposed to execute the combination of using multi-centered images DWT-PCA-KNN, taking the accompanying structure for integrating disparate and complementary information. Besides a prospect of a future ideal technique, medical terminology has been covered by the application of different medical procedures. Continuous wavelet transformation provides no means to use lossless compression because integer numbers are not transformed.

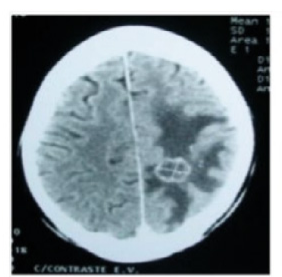

a) CT image

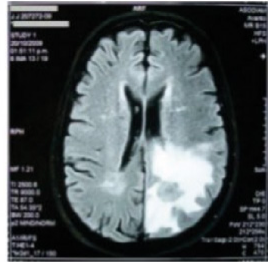

a) MRI image

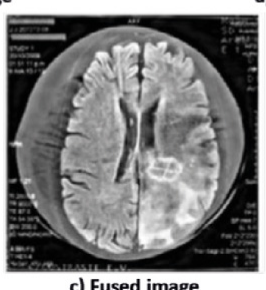

c) Fused image

Fig. 8. Result of fused brain CT and MRI images 
In the table 1 PSNR in the metric analysis is a quantitative evaluation of the results based on the Root Mean Square Error (RMSE). The value of PSNR should be higher

Table 1. Comparison of fused images

\begin{tabular}{|c|c|c|c|c|c|c|c|c|}
\hline \multirow{2}{*}{\multicolumn{2}{|c|}{ Patient }} & \multicolumn{7}{|l|}{ PSNR } \\
\hline & & $\mathrm{CT}$ & MRI & $\mathrm{CT}$ & MRI & $\mathrm{CT}$ & MRI & Fused Image \\
\hline Normal & $P_{\text {Ref }}$ & 12.4717 & 15.6641 & 0.9817 & 0.6836 & 0.2379 & 0.1647 & 5.0399 \\
\hline \multirow{3}{*}{ Abnormal } & P-1 & 13.7360 & 16.0250 & 0.9708 & 0.6297 & 0.2057 & 0.1580 & 3.4252 \\
\hline & $\mathrm{P}-2$ & 13.23011 & 17.0289 & 0.9042 & 0.7833 & 0.2180 & 0.1408 & 3.6517 \\
\hline & $P-3$ & 4.2156 & 16.9009 & 0.8877 & 0.7281 & 0.1946 & 0.1429 & 3.6474 \\
\hline
\end{tabular}

Source: own work

The results of the tests indicate that synthesized images have more detail and clarity than the pictures of the source after fusion. All the attributes of the two models are CT and MRI. Besides, Wavelet procedure is appropriate for multi-focused fusion image processing features at various levels [28].

\section{Conclusion}

In this paper, multi-modal fusions of image CT as well as MRI medical images using the fully mechanized wavelet transforming process in the MATLAB software have been considered. Reliably, the preservation of frequency and time of wavelet characteristic and natural improvement of PCA qualities enable this technique to fuse medical images. In the portrayal of spatial and spectral data, finally, fusion images gained from this combination method are polished as well as delicate brain tissue refinement. Therefore, in a separate fused image, it produces two distinct medical ways that support the motivation behind medical image fusion. The results are visually improved and a highly estimated image fusion can be obtained from the proposed therapeutic fusion technology formula. As defined in the high estimates of the fusion parameter, the test results show substantial improvements in data reform and quality characteristics of the acquired final image compared with the other pictures combined. The best-combined picture is distinct, easy to see, and experts can use it to analyze and recommend appropriate medicines. 


\section{Future scopes}

This research work has various options to further process with additional methods and algorithms as several studies in this field are underway. In this context, the basis for some proposed common experimental improvement is:

Multiple wavelets used for superior image quality and the clarification of multiwavelet productivity of image remote sensor fusion over common WT strategies. The equivalent can also be implemented and validated in future projects through the progress of image quality measurements and representations.

The pictures were not recorded in this work. Image accusations and recordings would increase the experimental performance since many unrecorded photos could be viewed as a source of input photos. It would also help to make sampling tests or quality images possible to evaluate the image fusion algorithms.

The quality of the image fusion has been analyzed concerning two images according to optical picture sets. The effectiveness of the fusion could be better evaluated if it is possible to do the same in several different variable models. However, this was not necessary since such a test set for variable images was not available.

\section{Acknowledgment}

The author would like to thank the editor-in-chief sincerely for his valuable suggestions for improvising this article. This study is not funded through grants.

\section{References}

1. H. Zheng, D.Z.Y. Hu, S. Li.,"Study on the Optimal Parameters of Image Fusion Based on Wavelet Transform [J]," Journal of Computational Information Systems, (2010) 131-137.

2. S.P. Yadav, S. Yadav, "Fusion of Medical Images in Wavelet Domain: A Discrete Mathematical Model,” IngenieríaSolidaría, vol. 14, no.25,2018, doi: https://doi.org/10.16925/.v14i0.2236

3. N. H. Ja'afar, "Medical Image Compression using Curvelet and Wavelet Transforms," Journal of Advanced Research in Dynamical and Control Systems, vol. 12, no. 4, Special Issue, pp. 15181523, 2020, https://doi.org/10.5373/jardcs/v12sp4/20201631 
4. S.P. Yadav, S. Yadav, "Fusion of Medical Images in Wavelet Domain: A Hybrid Implementation," Computer Modeling in Engineering \& Sciences, vol. 122, no. 1, 2020, pp. 303-32.[Online]. Available: https://www.techscience.com/CMES/v122n1/38247

5. S. Li, J. T. Kwok,Y. Wang, "Combination of images with diverse focuses using the spatial frequency," Information Fusion 2, pp. 169-176, 2001,doi: https://doi.org/10.1016/s1566-2535 (01)00038-0

6. A. S. Sekhar and M. N. G. Prasad, "A novel approach of image fusion on MR and CT images using wavelet transforms," 2011 3rd International Conference on Electronics Computer Technology, Apr. 2011, doi: https://doi.org/10.1109/icectech.2011.5941881

7. S. Kor and U. Tiwary, "Feature level fusion of multimodal medical images in lifting wavelet transform domain," The 26th Annual International Conference of the IEEE Engineering in Medicine and Biology Society, doi: https://doi.org/10.1109/iembs.2004.1403455.

8. A. H. Gunatilaka and B.A. Baertlein, "Feature-level and decision-level fusion of noncoincidently sampled sensors for land mine detection," IEEE Transactions on Pattern Analysis and Machine Intelligence, vol. 23, no. 6, pp. 577-589, Jun. 2001,doi:https://doi.org/10.1109/34.927459.

9. D. Kaur, P S Mann,"Medical Image Fusion Using Gaussian Filter, Wavelet Transform and Curvelet Transform Filtering," International Journal of Engineering Science \& Advanced Technology, vol. 4, no. 3, pp. 252-256.[Online]. Available: https://pdfs.semanticscholar. org/9bab/3547fd6d73d83e8760c6523a3f344d3435b7.pdf

10. M.C. El-Mezouar, N. Taleb, K. Kpalma, J.Ronsin, "An IHS-Based Fusion for Color Distortion Reduction and Vegetation Enhancementin IKONOS Imagery," IEEETransactionson Geo-science And Remote Sensing, vol. 49, no. 5, May 2011, doi: https://doi.org/10.1109/TGRS.2010.2087029

11. J. Bhardwaj, A, Nayak, "Haar wavelet transform-based optimal Bayesian method for medical image fusion,"Med BiolEngComput, 2020, doi: https://doi.org/10.1007/s11517-020-02209-6.

12. R. P. Singh Chauhan, R. Dwivedi, S.Negi,“'Comparative Evaluation of DWT and DT-CWT for Image Fusion and De-noising," International Journal of Applied Information Systems (IJAIS),vol. 4, no.2, September 2012. [Online]. Available: www.ijais.org,doi: https://doi.org/10.5120/ ijais $12-450662$

13. P. Ganasala, V. Kumar," $C T$ and MR image fusion scheme in non-sub sampled contourlet transform domain,” Jigit/maging, vol. 27, no. 3, pp. 407-418, doi: 10.1007/s10278-013-9664-X. 
14. K. V. Swathi, C.H.Bindu, "Modified Approach of Multimodal Medical Image Using Daubechies Wavelet Transform," International Journal of Advanced Research in Computer and Communication Engineering, vol. 2, no.11, November 2013. [Online]. Available: https://ijarcce. $\mathrm{com} /$ ?s=kanisetty+venkata+swathi\&et_pb_searchform_submit=et_search_proccess\&et_ pb_include_posts=yes

15. J. Srikanth, C.N Sujatha, "Image Fusion Based on Wavelet Transform for Medical Diagnosis," Int. Journal of Engineering Research and Applications, vol. 3, no. 6, Nov-Dec 2013, pp.252-256. [Online]. Available: https://www.ijera.com/papers/Vol3_issue6/A036247251.pdf

16. J. Bhardwaj, A.Nayak, "Discrete Wavelet Transform and Bird Swarm Optimized Bayesian Multimodal Medical Image Fusion,"Helix, vol. 10, no. 01, 2020,pp 07-12 7, doi: https://doi. org/10.29042/2020-10-1-07-12

17. K. R.Penmetsa, V.G.Prasad Naraharisetti, N.Venkata RAO, "An Image Fusion Technique ForColor Images Using Dual-Tree Complex Wavelet Transform," International Journal of Engineering Research \& Technology (IJERT), vol. 1,no. 8, October 2012. [Online]. Available: https://www. ijert.org/research/an-image-fusion-technique-for-colour-images-using-dual-tree-complexwavelet-transform-IJERTV1IS8642.pdf

18. G. J.Patil, S. Hasan, "Application of Image Fusion Using Wavelet Transform In Target Tracking System," International Journal of Engineering Research \& Technology (IJERT), vol. 1, no. 8, October 2012. [Online]. Available: https://www.ijert.org/research/application-of-image-fusion-using-wavelet-transform-in-target-tracking-system-IJERTV1IS8293.pdf

19. C.Pavithra, Dr. S. Bhargavi, "Fusion of Two Images Based on Wavelet Transform," International Journal of Innovative Research in Science, Engineering and Technology, vol. 2, no.5, May 2013. [Online]. Available: http://www.ijirset.com/upload/may/73_FUSION\%200F.pdf

20. R. Singh, A. Khare, "Multimodal medical image fusion using daubechies complex wavelet transform," Information \& Communication Technologies (ICT), 2013,pp. 869-873, doi:https:// doi.org/10.1109/cict.2013.6558217

21. G. Singh, T. K.Rawat, "Color Image Enhancement by Linear Transformations Solving out of Gamut Problem," International Journal of Computer Applications (USA), vol.6, no-14, pp.28-32, April 2013,doi: https://doi.org/10.5120/11464-7072

22. Ai. Deng, J. Wu, S. Yang, "An Image Fusion Algorithm Based on Discrete Wavelet Transform and Canny Operator," Advanced Research on Computer Education, Simulation and Modeling 
Communications in Computer and Information Science, vol. 175, 2011, pp 32-38, doi: https:// doi.org/10.1007/978-3-642-21783-8_6

23. N. Cvejic, D. Bull, N. Canagarajah, "Region-Based Multimodal Image Fusion Using ICA Bases," IEEE Sensors Journal, vol. 7, no. 5, pp. 743-751, May 2007,doi: https://doi.org/10.1109/ jsen.2007.894926

24. P. S. Sengar, T. K. Rawat,H. Parthasarathy, "Color image enhancement by scaling the discrete wavelet transform coefficients," 2013 Annual International Conference on Emerging Research Areas and 2013 International Conference on Microelectronics, Communications and Renewable Energy, Kanjirapally, 2013, pp. 1-6,doi: 10.1109/AICERA-ICMiCR.2013.6575994.

25. D. Aneja, T. K.Rawat, "Fuzzy Clustering Algorithms for Effective Medical Image Segmentation," International Journal of Intelligent Systems and Applications (Hong-Kong), 2013, vol. 11, 55-61, doi: 10.5815/ijisa.2013.11.06.

26. P.M.Pradnya, D. R. Sachin,"Wavelet based image fusion techniques," International Conference on Intelligent Systems and Signal Processing (ISSP), 2013, pp. 77-81,doi: https://doi. org/10.1109/issp.2013.6526878

27. H. Li, B. S. Manjunath, S. K. Mitra, "Multisensor Image Fusion Using the Wavelet Transform," Graphical Models and Image Processing, vol. 57, no. 3, pp. 235-245, May 1995, doi: https://doi. org/10.1006/gmip.1995.1022

28. S.P. Yadav, S. Yadav, "Image fusion using hybrid methods in multimodality medical images,"Medical \& Biological Engineering \& Computing, vol. 58, no. 4,2020,pp.1-19, doi: https://doi.org/10.1007/s11517-020-02136-6

29. Y. Na, M. Ehlers, W.Yang, L. Shi, "Adaptive remote sensing image fusion with multiwavelet transform,"Remote Sensing for Environmental Monitoring, GIS Applications, and Geology V, International Society for Optics and Photonics, 2005,pp. 5983-598302, doi: https://doi. $\operatorname{org} / 10.1117 / 12.627598$ 